\title{
Concurrence et antisélection multidimensionnelle en assurance
}

\author{
Bertrand VILLENEUVE *
}

RÉSUMÉ. - Le modèle standard de concurrence avec antisélection en assurance (Rothschild et Stiglitz) suppose que les types ne diffèrent que par leur probabilité d'accident. Supposant qu'ils puissent aussi différer par leur attitude face au risque, nous mettons en évidence des configurations inhabituelles : des équilibres multiples; des profits strictement positifs; de l'assurance aléatoire. Nous caractérisons les différents régimes d'équilibre possibles et analysons les paramètres qui les déterminent.

\section{Competitive Insurance Markets with Multidimensional Adverse Selection}

\begin{abstract}
In the Rothschild and Stiglitz model, assuming differences in risk aversions may lead to unusual equilibrium configurations like multiple equilibria, equilibrium positive profits, or random contracts. We characterize the various types of equilibria and give results on the determinants of the equilibrium regime. We conclude with a few remarks on classical equilibrium concepts in insurance economics, and we suggest policy implications.
\end{abstract}

\footnotetext{
* B. Villeneuve : Université de Toulouse (CEA, IDEI, LEERNA). Je remercie Alberto BennaRdo, Arnold ChASSAGnon, Pierre-André ChiapPoRI, Georges DIONNE, Isaac MEILIJSON et François PANNEQUIN pour les discussions utiles que j'ai pu avoir avec eux. Toutes les erreurs me sont imputables. J'ai bénéficié d'un soutien financier du CNRS au DELTA et de I'INSEE au CREST.
} 


\section{Introduction}

Les études de l'antisélection en assurance restent en général dans l'hypothèse que lors de la constitution de classes de risques en vue de réduire l'asymétrie d'information entre assureurs et assurés, seule la probabilité de sinistre reste pour les assureurs imparfaitement dévoilée. Pourtant, les différences résiduelles entre assurés pourraient être plus complexes et concerner aussi l'attitude générale face au risque. Le travail que nous présentons ici se penche sur l'enrichissement que permet l'hypothèse d'hétérogénéité des goûts lorsqu'elle est combinée à l'habituelle hétérogénéité des risques. L'hétérogénéité multidimensionnelle est abordée dans un cadre concurrentiel, ce qui, comparé aux travaux de Rochet et ChONÉ [1998] ou ARMSTRONG [1996] en monopole, simplifie l'analyse puisque les subventions croisées disparaissent nécessairement.

Dans une économie apparemment très homogène, ce type de situation émerge naturellement. Les chocs sur le revenu et l'épargne inobservable rendent possibles des évolutions très contrastées de l'aversion pour un risque donné ${ }^{1}$. Dans le même ordre d'idée, des agents de préférences identiques pourraient subir des risques assurables différents, mais aussi, de manière inobservable, des risques inassurables différents (background risk au sens de DoHERTY et SCHLESINGER [1983]). L'utilité apparente pour les assureurs du risque assurable devient ipso facto spécifique au type de l'agent ${ }^{2}$.

Notre étude pose la question de la robustesse des conclusions de RothSCHILD et STIGLITZ [1976, RS dans la suite] sur les plans des conséquences testables (l'allure des contrats), de la modélisation de la concurrence (le concept d'équilibre), ou sur le plan normatif (existence, efficacité). Dans un modèle inconfortable où nous renonçons à la propriété de croisement unique des courbes d'indifférence (PCU) ${ }^{3}$, sur laquelle s'appuient bien des raisonnements, nous proposons une caractérisation des régimes possibles à l'équilibre, ainsi qu'une analyse précise des conditions sur les paramètres du modèle (préférences, risques, distribution des types) qui déterminent le régime effectivement en vigueur. Trois entrangetés retiennent l'attention : la multiplicité, les profits positifs, et les contrats aléatoires.

Dans tous les cas, tout se joue autour du contrat destiné aux assurés qui subissent l'antisélection, les bas risques. Il faut d'abord se convaincre que les assureurs, en concurrence les uns avec les autres, cherchent à leur donner le meilleur contrat possible, sous la contrainte que les hauts risques ne doivent

1. On peut voir CHIAPPORI et alii [1994] pour un développement de ces idées dans un contexte d'aléa moral répété. LAFFONT et ROCHET [1988] montrent que des différences d'aversion pour le risque peuvent être exploitées pour élaborer efficacement des contrats d'assurance vie où l'assurance stricto sensu est mêlée à l'épargne de plusieurs manières.

2. Le cas où des risques différents sont assurés par des assureurs différents est étudié en concurrence par Fluet et PANNEQUin [1996] et en concurrence monopolistique par KoEHL et VilLENEUVE [2001].

3. En général, dans les modèles principal-agent, c'est une hypothèse explicite. Voir, par exemple, SPENCE [1974] ou GUESNERIE et LAFFONT [1984]. 
pas adopter la proposition. Dans le modèle de RS, cela implique que, alors que les hauts risques reçoivent une assurance complète, et actuarielle (c'est-àdire telle que la prime vaut l'espérance de l'indemnité), les bas risques ne peuvent recevoir qu'une assurance partielle, actuarielle elle aussi.

Si les types diffèrent par leurs aversions pour le risque, on introduit la propriété que (si l'on nous permet un abus de langage) leurs taux de substitution entre « espérance de la richesse » et «variance de la richesse » diffèrent. Nous expliquons comment cela peut être exploité à l'équilibre, en prenant pour point de référence le meilleur contrat à profit nul pour le bas risque prévu par le modèle de RS.

Si le bas risque est plus riscophobe que le haut risque, il est prêt à plus de sacrifice (plus faible espérance) pour obtenir une assurance de meilleure qualité (plus faible variance), or un tel contrat réalise nécessairement un profit positif. Comment cela peut-il être stable ? Dans le cas le plus simple, tous les assureurs voudraient assurer les bas risques, mais un assureur doit se charger d'assurer les hauts risques pour que l'équilibre se fasse. Cet assureur ne peut pas dévier, car il cesserait d'offrir le contrat (celui des hauts risques) qui rend possibles les profits sur les bas risques, détruisant lui-même ses raisons de dévier.

$\mathrm{Si}$ le bas risque est moins riscophobe, on peut envisager de mettre du bruit dans l'indemnité et d'augmenter l'espérance de cette indemnité. La difficulté est de trouver une perturbation qui ne soit pas attractive pour le haut risque (plutôt riscophobe), mais qui le soit pour le bas risque.

Les intuitions précédentes sont correctes pour comprendre les conditions nécessaires pour un certain régime. C'est l'approche retenue par ARNOTT et STIGLITZ [1988] pour l'assurance aléatoire ou dans plusieurs travaux indépendants (Chassagnon [1996], SMART [2000], Villeneuve [1996] ou WAMBACH [1997]) pour les profits positifs. Cependant, l'analyse est incomplète en l'absence de conditions suffisantes. Notre article traite de toutes les possibilités théoriques et propose des caractérisations plus fines et des résultats de statique comparative. Nous traitons aussi de l'intervention publique dans les situations étudiées.

SMART [2000], par exemple, suppose des technologies d'assurance nonconvexes et rationalise ainsi de manière élégante l'équilibre de RILEY [1979]. Il montre l'unicité de l'équilibre. Nous présentons deux formes de multiplicité interdites par son approche : d'abord, parce que le nombre d'assureurs n'est pas nécessairement infini, nous montrons que le contrat des hauts risques peut aussi être profitable. Par ailleurs, il concentre l'attention sur les allocations séparatrices et les allocations mélangeantes. Nous montrons que les hauts risques peuvent se mélanger partiellement aux bas risques dans le contrat destinés à ces derniers, et ceci parce que des contrats profitables peuvent tolérer une proportion d'assurés coûteux. D'autre part, nous ne supposons pas a priori que le bas risque est uniformément plus riscophobe. La statique comparative de l'aversion pour le risque nous permet notamment d'envisager, et d'exclure sous une condition originale, l'assurance aléatoire.

L'approche qui est adoptée vise à clarifier le rôle critique des paramètres du modèle de RS, c'est-à-dire dans quelle mesure ils déterminent, toutes choses égales d'ailleurs, les propriétés d'un équilibre. On pourrait résumer ainsi : les proportions des différents types d'agents sont critiques pour l'existence et 
l'optimalité de l'équilibre (RS) ; l'hétérogénéité des pertes espérées est critique pour la séparation des types (RS, FAGART [1996]) ; l'hétérogénéité des attitudes face au risque est critique pour le profit des assureurs, et pour l'existence. Le concept d'équilibre, quel qu'il soit, est critique pour la soutenabilité des profits.

Des travaux récents (De Meza et Webb [2000], Jullien, Salanié et Salanié [2000]) étudient l'antisélection sur l'aversion pour le risque combinée à l'aléa moral. Il y est montré que les plus riscophobes tendent à présenter un risque (endogène) plus faible à contrat donné. Bien que ce résultat ne soit pas nécessairement conservé à l'équilibre, il suggère qu'un modèle qui incorporerait aléa moral, antisélection sur l'aversion pour le risque et sur le risque accorderait une place particulièrement importante au cas du bas risque plus riscophobe, à l'origine des résultats les plus éloignés de Rothschild et Stiglitz dans notre étude.

La section 2 présente le modèle et rappelle les résultats du cas particulier de Rothschild et Stiglitz. La section 3 présente une caractérisation générale des équilibres avec antisélection sur l'aversion pour le risque et le risque. Des résultats de statique comparative et la généralisation multidimensionnelle sont donnés. La section 4 discute du rôle de l'assurance aléatoire, et donne une condition de non-coexistence avec des profits positifs à l'équilibre. La section 5 discute le concept d'équilibre et conclut.

\section{Le modèle}

Les consommateurs possèdent un patrimoine de valeur $W$ et risquent de perdre un bien de valeur $d$. Ils peuvent être de deux types : le haut risque $H$ subit le dommage avec probabilité $p_{H}$, tandis que le bas risque $L$ le subit avec probabilité $p_{L}$, où $p_{L}<p_{H}$. Les types ne sont pas supposés présenter la même attitude face au risque et leurs fonctions d'utilité de Von NeumannMorgenstern sont notées $u_{H}$ et $u_{L}$. Les indices absolus d'aversion pour le risque de ces deux fonctions, notés $a_{H}$ et $a_{L}$, sont supposés positifs ou nuls et continus. Nous supposons également que les courbes d'indifférence des deux types ne se croisent qu'un nombre fini de fois. La section 3.3 traite le cas où risque et préférences ne sont pas parfaitement corrélés, ce qui autorise quatre types d'assurés.

Le jeu de concurrence à la Bertrand est le suivant : 1) chaque assureur offre un contrat d'assurance et un seul, spécifiant la prime et l'indemnité ; les assureurs ne peuvent pas faire de discrimination et doivent accepter tout client désireux de souscrire cette offre ; 2) les assurés choisissent optimalement une offre parmi les offres disponibles. Les assureurs sont au nombre de $N \geqslant 3$, afin de minimiser les possibilités de collusion tacite : en effet, chaque assureur n'offrant qu'un contrat, l'un pourrait se spécialiser sur les bas risques et l'autre sur les hauts risques; les niveaux de profits seraient alors peu contraints (et notamment pourraient être strictement positifs sans difficulté), puisque la déviation de l'un entraînant le mouvement de toute sa clientèle, les incitations à dévier sont efficacement bloquées. L'introduction 
d'un troisième assureur (plus d'assureurs que de types) permet une concurrence plus active. Nous verrons cependant que des profits positifs peuvent apparaître quand même.

Le problème peut être symbolisé, à des fins de statique comparative, par $\mathcal{P}\left[N, p_{H}, u_{H}, \lambda_{H}, p_{L}, u_{L}, \lambda_{L}, W, d\right]$ où $\lambda_{K}$ est le nombre normalisé d'agents de type $K=H, L\left(\lambda_{H}+\lambda_{L}=1\right)$. Pour simplifier l'exposition, les résultats présentés supposent dans un premier temps que les contrats offerts sont déterministes. Les intuitions principales ne sont pas affectées par cette restriction. La section ?? explique le rôle potentiel de l'assurance aléatoire, donne sa structure et ses limites.

Si le type de l'individu était observable, la concurrence entre assureurs jouerait pleinement, et chaque type recevrait une assurance complète actuarielle, que l'on note $\alpha_{H}^{*}$ et $\alpha_{L}^{*}$. Le remboursement est $d$, mais la prime varie avec le type, soit $p_{H} d$ et $p_{L} d$. Cette allocation n'est plus réalisable dès lors que le type d'un individu n'est connu que de lui-même, comme nous le supposons ici, puisque tous préféreraient l'assurance du bas risque, moins chère.

En supposant que les assureurs connaissent la distribution des types, nous cherchons les équilibres de Nash en stratégies pures. Chaque assureur anticipe les offres faites par ses concurrents, et l'autosélection des agents, il anticipe donc correctement les caractéristiques de sa clientèle. En espérance, son profit doit être positif ou nul.

Dans toutes les figures, l'abscisse $W_{1}$ désigne la consommation en l'absence d'accident et l'ordonnée $W_{2}$ la consommation en cas d'accident. En l'absence d'assurance, l'individu consomme $(W, W-d)$, et tout point $\left(W_{1}, W_{2}\right)$ peut être vu comme un contrat d'assurance déterministe. Ainsi, la droite à $45^{\circ}$ représente le lieu des assurances complètes. Les droites d'isoprofit ont pour équation, respectivement, $\left(1-p_{H}\right)\left(W-W_{1}\right)+$ $p_{H}\left(W-d-W_{2}\right)=\pi_{H}$, et $\left(1-p_{L}\right)\left(W-W_{1}\right)+p_{L}\left(W-d-W_{2}\right)=\pi_{L}$, où $\pi_{H}$, par exemple, représente le profit réalisé en espérance par le contrat s'il est adopté par $H$. Les courbes d'indifférence des consommateurs présentent toutes un contour supérieur convexe.

Si $u_{H}=u_{L}$, on entre dans le cadre de l'article de Rothschild et Stiglitz :

1. Le haut risque reçoit $\alpha_{H}^{*}$, pleine assurance au prix actuariellement juste.

2. Le bas risque reçoit une assurance partielle (l'indemnité est plus faible que la perte) actuariellement juste ; cette assurance est aussi bonne pour $H$ que son assurance complète. On dit que le haut risque fait peser sa contrainte d'autosélection sur le bas risque.

3. Il existe un équilibre en stratégie pure si et seulement si la proportion des bas risques $\lambda_{L}$ est assez faible, la proportion frontière $\lambda_{L}^{\text {exist }}$ étant strictement comprise entre 0 et $1^{4}$.

4. L'allocation d'équilibre est efficace sous contrainte d'information si et seulement si la proportion des bas risques $\lambda_{L}$ est assez faible, la proportion frontière $\lambda_{L}^{\text {eff }}$ étant strictement comprise entre 0 et $\lambda_{L}^{\text {exist }}$.

4. Rappelons le schéma de démonstration. Tout équilibre en stratégie pure est nécessairement séparateur, et satisfait aux conditions 1 et 2 . Or, si $\lambda_{L}$ est assez grand, un contrat mélangeant (attirant tous les types) peut être trouvé qui domine au sens de Pareto l'allocation testée. Il n'y a donc plus d'équilibre. 


\section{L'équilibre de Rothschild et Stiglitz}

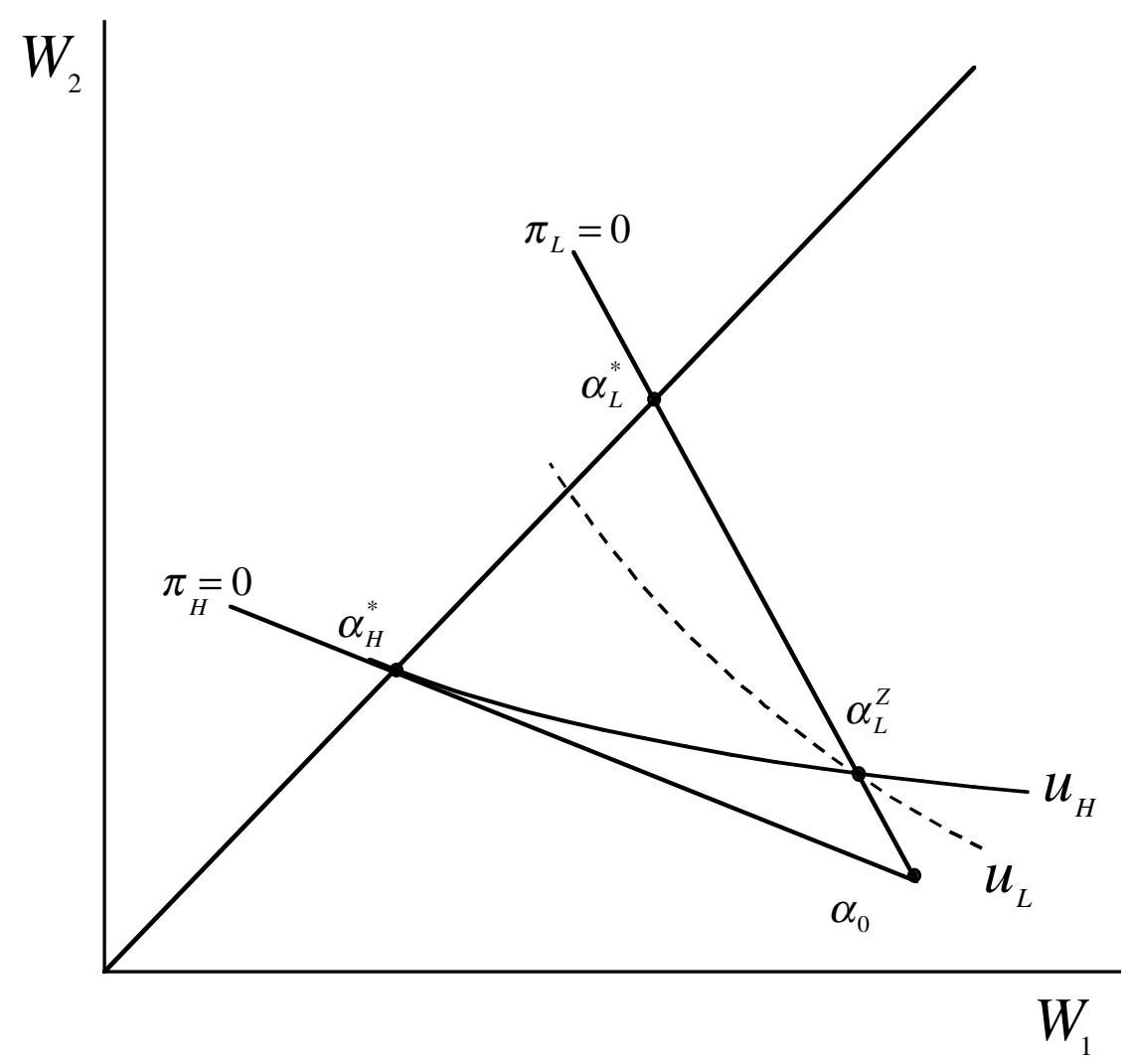

La figure 1 donne une synthèse des résultats. $\pi_{H}=0$ et $\pi_{L}=0$ représentent les droites actuarielles des types $H$ et $L$ respectivement ; $u_{H}$ et $u_{L}$ représentent des courbes d'indifférence. L'allocation sans assurance est notée $\alpha_{0}$.

Notons $\alpha_{L}^{Z}$ (Z pour un profit valant zéro) l'intersection entre la courbe d'indifférence du haut risque passant par $\alpha_{H}^{*}$ et la droite actuarielle du bas risque. C'est le seul contrat satisfaisant les conditions 1 et 2. Le résultat de RS repose notamment sur le fait que la disposition marginale à payer de $H$ pour un supplément d'assurance est en tout point supérieure à celle de $L$, autrement dit, les courbes d'indifférence des deux types se croisent toujours de la même façon. Supposons que cette propriété puisse être localement violée (à cause de l'aversion pour le risque plus grande du bas risque), de sorte qu'en $\alpha_{L}^{Z}$, la pente de la courbe d'indifférence du bas risque soit plus faible en valeur absolue que celle du haut risque (voir figure 2). $\left(\alpha_{H}^{*}, \alpha_{L}^{Z}\right)$ n'est plus un équilibre puisqu'un assureur peut attirer toute la clientèle des bas risques en réalisant un profit positif, il lui suffit d'offrir $\alpha_{L}$ dans la zone hachurée de la figure. A partir de cette idée, nous montrons la compatibilité d'un équilibre concurrentiel avec la réalisation de profit. 
FIGURE 2

\section{Profit positif}

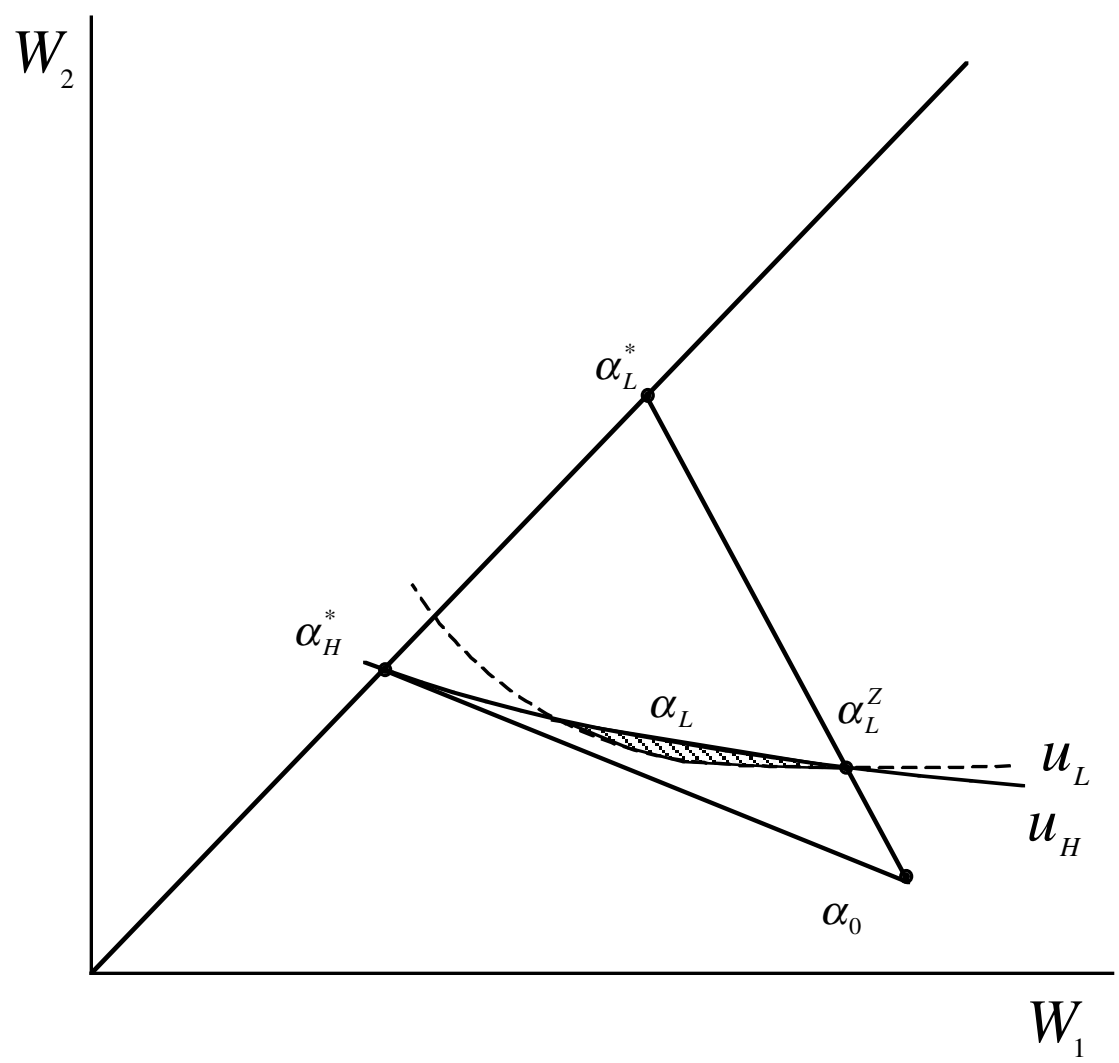

\section{Les équilibres}

\subsection{Caractérisation générale}

On peut sans perte de généralité concentrer l'attention sur les contrats effectivement pris à l'équilibre par une fraction des agents. En effet, le retrait d'un contrat inactif (stratégie faiblement dominée) n'augmente pas les possibilités de déviation des assureurs actifs, les assurés adoptant toujours le(s) meilleur(s) contrat(s) disponible(s).

\section{Proposition 1. À l'équilibre}

1. Deux contrats différents au moins sont offerts.

2. Il existe un contrat et un seul qui n'est choisi que par H. C'est un contrat d'assurance complète que l'on note $\alpha_{H}$. 
3. Tous les contrats autres que $\alpha_{H}$, notés $A_{L}=\left\{\alpha_{L}^{i}\right\}_{i \in G}$ (G étant un ensemble fini et minimal d'indices), sont pris par une fraction des $L$.

\section{4. $H$ est indifférent entre tous les contrats.}

PreUVE : 1. Si un seul contrat est offert, la concurrence oblige qu'il soit optimal au sens de Pareto parmi les contrats faisant un profit nul lorsqu'ils rassemblent tous les assurés. Ainsi l'un des types refuserait moins d'assurance au prix implicite (risque moyen), et l'autre refuserait plus d'assurance à ce prix. Or les taux marginaux de substitution des deux types ne peuvent pas être égaux en un tel optimum de Pareto (ils peuvent l'être en un autre contrat) : au prix moyen, l'optimum de $H$ est une sur-assurance stricte, et l'optimum de $L$ est une sous-assurance stricte. Cette différence des TMS peut être exploitée par un assureur déviant, offrant un contrat n'attirant que les bas risques tout en réalisant un profit positif. L'équilibre s'effondre, le contrat initial devenant déficitaire.

2. Raisonnons par l'absurde, et supposons qu'aucun contrat pris par $H$ n'est une assurance complète. L'assureur offrant un contrat pris par $H$ peut compléter l'assurance (inefficace) qu'il propose et donc attirer tous les $H$ tout en réalisant un profit légèrement supérieur sur tous les types (il ne craint pas d'attirer $L$, forcément profitable sur un contrat d'assurance complète profitable sur $H$ ). On obtient ainsi qu'un contrat d'assurance complète est toujours offert (il est unique, tous les contrats d'assurance complète étant classés sans ambiguïté).

Supposons maintenant que le contrat d'assurance complète, noté $\alpha_{H}$, attire aussi une fraction des $L$. $L$ a en $\alpha_{H}$ une disposition marginale à payer pour l'assurance inférieure à celle de $H$ (seules comptent, au premier ordre, les probabilités, autour des assurances complètes). Il existe donc un contrat offrant légèrement moins d'assurance que $\alpha_{H}$, aussi bon pour $H$, mais meilleur pour $L$, que $\alpha_{H}$. Les profits sur $H$ sont les mêmes au premier ordre, mais les profits sur $L$ sont supérieurs. D'autre part, l'offre attire tous les $L$. Cette offre est donc plus profitable, au premier ordre, pour l'assureur. Par continuité, on peut trouver dans le voisinage de ce contrat une offre donnant une utilité supérieure à tous les types, tout en laissant un profit supérieur à l'assureur (amélioration au sens de Pareto). Enfin, $G$ est fini en raison de l'hypothèse selon laquelle les courbes d'indifférence des deux types ne se coupent jamais un nombre infini de fois.

3 . et 4 . D'après 2 , tous les contrats de $A_{L}$ doivent être adoptés par une fraction des $L$, donc sont équivalents pour $L$. Si l'un des contrats de $A_{L}$ est strictement inférieur à $\alpha_{H}$ pour $H$, alors seul $L$ peut l'adopter à l'équilibre. Ce contrat n'étant pas optimal pour $L\left(\alpha_{L}^{*}\right.$ est inaccessible), et $H$ ne faisant pas peser de contrainte d'autosélection sur lui, il peut être amélioré.

La question reste de savoir par qui. Si le nombre d'assureurs offrant $\alpha_{H}$ est supérieur ou égal à deux, alors $\alpha_{H}$ donne un profit nul. (Sinon, en effet, l'un de ces assureurs pourrait attirer tous les $H$ en baissant très légèrement le prix, obtenant un profit supérieur grâce à l'augmentation discrète de la clientèle.) Or un assureur réalisant un profit nul est prêt à dévier pour réaliser un profit sur les $L$. S'il n'y a qu'un seul assureur offrant $\alpha_{H}$, alors deux au moins (puisque $N \geqslant 3$ ), assurent les $L$. L'assureur offrant le contrat non optimisé de $L$ peut augmenter son profit en déviant (gain d'efficacité). Dans 
tous les cas de figure envisagés, il existe un déviant. En conséquence, tous les contrats de $A_{L}$ et $\alpha_{H}$ sont nécessairement équivalents pour $H$.

Il ressort de cette proposition que, si $\alpha_{H}$ est un contrat d'équilibre, l'ensemble $A_{L}$ des contrats offerts à $L$ est non vide et contenu dans l'ensemble $A_{L}^{*}\left(\alpha_{H}\right)$ que nous définissons comme l'ensemble des contrats maximisant l'utilité des $L$ sous la contrainte d'autosélection des $H$ et sous la contrainte de profit positif ou nul des assureurs. Par ailleurs, il apparaît dans la démonstration d'une part que le nombre d'assureurs offrant un contrat joue un rôle, et, d'autre part, qu'une fraction des $H$ peut adopter certains contrats de $A_{L}$. Un équilibre est donc un ensemble détaillé de données, notamment :

- $\left(\alpha_{H},\left\{\alpha_{L}^{i}\right\}_{i \in G}\right)$, un ensemble de contrats où $\alpha_{H}$ est un contrat d'assurance complète profitable compatible avec la participation de $H$, et où $\alpha_{L}^{i} \in A_{L}^{*}\left(\alpha_{H}\right)$.

- $N_{H}$ et $N_{L}^{i}$, respectivement, le nombre d'assureurs qui offrent $\alpha_{H}$ et $\alpha_{L}^{i}$.

- Une répartition des assurés entre offres équivalentes, soit $\lambda_{H}^{0}$ individus de type $H$ sur $\alpha_{H}$, et $\lambda_{H}^{i}$ (respectivement $\lambda_{L}^{i}$ ) individus de type $H$ (respectivement $L) \operatorname{sur} \alpha_{L}^{i}$.

Les profits par tête de $\alpha_{H}$ sur $H$, de $\alpha_{L}^{i}$ sur $H$ et de $\alpha_{L}^{i}$ sur $L$ sont notés respectivement $\pi_{H}, \pi_{H}^{i}$ et $\pi_{L}^{i}$. Pour simplifier, nous faisons l'hypothèse que les assurés se répartissent de manière équiprobable entre les assureurs offrant le même contrat.

Un autre aspect fondamental d'un tel équilibre est le comportement (horséquilibre, et tel qu'il est anticipé par les assureurs) des individus « voyant leur contrat disparaître ». Par exemple, si un assureur est le seul à offrir un contrat, il doit anticiper les mouvements des assurés qu'il délaisse en déviant. Pour éviter un excès de complications, nous proposons la règle suivante, donnant des inégalités simples dans le théorème, sans perdre les aspects essentiels : en cas de disparition d'un contrat, les assurés suivent leur assureur si cela est un choix non dominé (ce n'est en général pas le seul).

\section{THÉORÈME 1. 1. Tout équilibre vérifie les relations suivantes} $\forall i, i^{\prime}$ :

(1) si $N_{L}^{i}=1: \lambda_{H}^{i} \pi_{H}^{i}+\lambda_{L}^{i} \pi_{L}^{i} \geqslant \frac{\left(\lambda_{H}^{i^{\prime}}+\lambda_{H}^{i}\right) \pi_{H}^{i^{\prime}}+\left(\lambda_{L}^{i^{\prime}}+\lambda_{L}^{i}\right) \pi_{L}^{i^{\prime}}}{N_{L}^{i^{\prime}}+1}$;

$$
\begin{gathered}
\text { si } N_{L}^{i} \geqslant 2: \frac{\lambda_{H}^{i} \pi_{H}^{i}+\lambda_{L}^{i} \pi_{L}^{i}}{N_{L}^{i}} \geqslant \frac{\lambda_{H}^{i^{\prime}} \pi_{H}^{i^{\prime}}+\lambda_{L}^{i^{\prime}} \pi_{L}^{i^{\prime}}}{N_{L}^{i^{\prime}}+1} ; \\
\frac{\lambda_{H}^{i} \pi_{H}^{i}+\lambda_{L}^{i} \pi_{L}^{i}}{N_{L}^{i}} \geqslant \lambda_{H} \pi_{H} ; \\
\text { si } N_{H}=1: \lambda_{H}^{0} \pi_{H} \geqslant \frac{\left(\lambda_{H}^{0}+\lambda_{H}^{i}\right) \pi_{H}^{i}+\lambda_{L}^{i} \pi_{L}^{i}}{N_{L}^{i}+1} ; \\
\text { si } N_{H} \geqslant 2: \pi_{H}=0 \text { et } \pi_{L}^{i}=0 .
\end{gathered}
$$


2. S'il existe un contrat d'équilibre strictement profitable, tout assureur est actif.

3. Si $\pi_{H}=0$, alors une allocation respectant les conditions précédentes est un équilibre si et seulement si la proportion des bas risques est assez faible.

PREUVE : 1. Les conditions assurent qu'un assureur offrant un contrat d'équilibre n'a pas intérêt à offrir un autre contrat d'équilibre. Ainsi, un assureur offrant $\alpha_{L}^{i}$ n'a pas intérêt à offrir $\alpha_{L}^{i^{\prime}}$ (équations 1 et 2) ni $\alpha_{H}$ (équation 3), et un assureur offrant $\alpha_{H}$ n'a pas intérêt à offrir $\alpha_{L}^{i}$ (équation 4 et 5). Dans (1), (2) et (4), l'assureur prend en compte le fait qu'il devra partager le profit, en se comptant lui-même. Les membres de droite des équations (1) et (4) tiennent compte du fait qu'un contrat disparaît, et que le déviant est «suivi»par son «ancienne » clientèle. Dans l'équation (3), la même expression rend compte de deux situations. Soit $\pi_{H}=0$, et la condition est évidemment nécessaire ; soit $\pi_{H}>0$, et le déviant peut faire mieux que partager la clientèle des $H$ avec les assureurs spécialisés dans les $H$ : il peut offrir un contrat d'assurance complète choisi arbitrairement proche de $\alpha_{H}$, mais plus avantageux pour les $H$ (donc les attirant tous), réalisant ainsi un profit supérieur. L'équation (5) dit simplement que les assureurs des $H$ peuvent être nombreux à la seule condition qu'aucun contrat, de toute façon, ne soit profitable.

2. Un assureur a toujours intérêt à offrir le contrat profitable plutôt que de faire une offre qui n'est pas prise.

3. En complément de 1, il reste à trouver les conditions sous lesquelles une offre extérieure à l'ensemble $\left(\alpha_{H},\left\{\alpha_{L}^{i}\right\}_{i \in G}\right)$ ne peut pas être offerte. Une telle déviation attire nécessairement tout le monde (elle ne peut pas attirer les seuls $L$, cela contredirait l'optimalité sous contrainte $\operatorname{des} \alpha_{L}^{i}$, et attirer sélectivement $H$ est exclu : $\pi_{H}=0$, donc aucune offre supérieure pour $H$ n'est envisageable). Le contrat déviant doit donc être profitable sur l'ensemble des assurés. Clairement, si un tel contrat existe pour une certaine proportion de $L$, alors il existe nécessairement pour une proportion supérieure. D'où le principe d'un seuil. Ce seuil existe nécessairement entre 0 et 1 , puisque si $\lambda_{L}$ est assez proche de 1 , il suffit de proposer un contrat à profit nul, d'assurance complète sur l'ensemble de la population, pour attirer tout le monde, et donc casser l'équilibre. D'autre part si $\lambda_{L}$ est assez proche de 0 , une telle offre est impossible, puisque tout contrat mélangeant profitable est alors proche de $\alpha_{H}$, lui-même dominé par les $\alpha_{L}^{i}$ pour $L$.

Ce raisonnement n'est pas possible dans sa totalité lorsque $\pi_{H}>0$, notamment parce qu'on ne peut plus exclure qu'une déviation attirant tous les types soit profitable sur tous. Voir cependant le corollaire 1.

Admettons que si la logique en est simple, il est difficile de se représenter cet ensemble. Les trois cas particuliers suivants sont remarquables. 
Corollaire 1. Si $N=+\infty$, alors $\pi_{H}=0$, et toute valeur de $\lambda_{L}$ assez proche de zéro assure l'existence.

En effet, soit la condition (5) s'applique, soit $N_{H}=1$ et donc un contrat destiné à $L$ est offert par une infinité d'assureurs (en effet, $G$ est fini), auquel cas la condition (3) aboutit à la même conclusion : $\pi_{H}=0$.

Corollaire 2. Si pour tout $i, \pi_{L}^{i}=0$, alors nécessairement $N_{H} \geqslant 1$, $N_{L}^{i} \geqslant 1, \lambda_{H}^{0}=\lambda_{H}, \lambda_{H}^{i}=0$ et $\lambda_{L}^{i}>0$.

On a pratiquement la situation de RS, à ceci près que les bas risques peuvent (à ce niveau de généralité) se répartir entre plusieurs contrats qu'ils sont seuls à prendre au lieu d'un seul. Remarquons que certains assureurs peuvent être inactifs, ce qui n'est plus vrai dans le cas suivant.

\section{| Corollaire 3. Si pour tout $i, \pi_{L}^{i}>0$, alors $N_{H}=1$ et $\sum_{i} N_{L}^{i}=N-1$.}

C'est l'équilibre à profit positif. Le seul assureur offrant $\alpha_{H}$ ne doit pas avoir intérêt à dévier vers un contrat destiné à $L$. S'il le faisait, $\alpha_{H}$ ne serait plus offert, et le contrat qu'il offre en déviation doit donc être profitable lorsqu'il rassemble tous les assurés. Or si un tel contrat existait, l'équilibre lui-même n'existerait pas.

Ce cas illustre bien les deux dimensions dans la multiplicité des équilibres. L'une porte sur le partage des profits entre $\alpha_{H}$ et $\alpha_{L}^{i}$. Cette multiplicité est liée au nombre d'assureurs, et disparaît pour $N$ vers $+\infty$, car le seul contrat possible pour $H$ est alors $\alpha_{H}^{*}$, dont le profit est nul. L'autre multiplicité vient de ce que les $H$ peuvent se distribuer entre $\alpha_{H}$ et $\alpha_{L}^{i}$. Il est utile de rappeler que dans le modèle original de $\mathrm{RS}$, le fait que les types soient parfaitement séparés n'est pas une hypothèse, mais fait partie intégrante des conditions d'équilibre. Ici, cette séparation n'est pas nécessaire. Toutefois, les $H$ ne doivent pas être représentés dans $\alpha_{L}^{i}$ au-delà de la proportion qui annule la profitabilité de $\alpha_{L}^{i}$.

Sur la stabilité de telles allocations, la remarque 1 plus bas donne une intuition supplémentaire.

\subsection{Statique comparative}

Dans toute la suite, nous supposerons que $N=+\infty$, afin de lever l'imprécision sur $\alpha_{H}$, valant maintenant nécessairement $\alpha_{H}^{*}$, pour nous concentrer sur les offres faites à $L$.

DÉFINITION 1. Nous appellerons situation $S$ le cas où un élément de $A_{L}^{*}\left(\alpha_{H}^{*}\right)$ donne un profit strictement positif sur les bas risques. Sinon nous parlerons de situation normale. 
Remarquons que dans la situation normale, les contrats d'équilibre sont ceux décrits par Rothschild et Stiglitz, soit $\alpha_{H}^{*}$ et $\alpha_{L}^{Z}$.

Nous proposons de rechercher des résultats de statique comparative qualitative. Supposons qu'un certain nombre de paramètres du programme $\mathcal{P}$ soient donnés, est-il possible d'obtenir la situation $S$ en balayant l'espace des paramètres libres?

La construction d'exemples de situation $S$ est facile (on fixe tout les paramètres sauf $u_{L}$, puis on prend assez d'aversion pour le risque) et utile à l'illustration mais n'est pas très satisfaisante d'un point de vue analytique puisqu'elle dit peu sur la dépendance du résultat d'équilibre aux paramètres fondamentaux autres que la fonction d'utilité du bas risque.

La situation $S$ est fondée sur une violation de la propriété de croisement unique (PCU) qui peut être caractérisée de la façon suivante : il existe au moins un point $\left(W_{1}, W_{2}\right)$ tel que la valeur marginale de l'assurance est plus élevée pour la bas risque que pour le haut risque. Pour être plus précis, le domaine de consommation est partagé :

$$
\begin{aligned}
& I^{+}=\left\{\left(W_{1}, W_{2}\right) \mid \frac{p_{L}}{1-p_{L}} \frac{u_{L}^{\prime}\left(W_{2}\right)}{u_{L}^{\prime}\left(W_{1}\right)} \leqslant \frac{p_{H}}{1-p_{H}} \frac{u_{H}^{\prime}\left(W_{2}\right)}{u_{H}^{\prime}\left(W_{1}\right)}\right\} \\
& I^{-}=\left\{\left(W_{1}, W_{2}\right) \mid \frac{p_{L}}{1-p_{L}} \frac{u_{L}^{\prime}\left(W_{2}\right)}{u_{L}^{\prime}\left(W_{1}\right)} \geqslant \frac{p_{H}}{1-p_{H}} \frac{u_{H}^{\prime}\left(W_{2}\right)}{u_{H}^{\prime}\left(W_{1}\right)}\right\}
\end{aligned}
$$

Dans $I^{+}$(resp. $I^{-}$), les bas risques ont une propension marginale à payer leur assurance plus faible (resp. plus forte) que les hauts risques. $T=I^{+} \cap I^{-}$est l'ensemble où les courbes d'indifférence des deux types sont tangentes. La droite d'assurance complète est incluse dans $I^{+}$, qui par conséquent n'est jamais vide. Remarquons également que la continuité de l'utilité marginale assure que si $I^{-}$n'est pas vide, alors $T$ n'est pas vide non plus. Enfin, si le bas risque est moins riscophobe, alors $I^{-}$et donc $T$ sont vides sous la droite à $45^{\circ}$. La figure 3 donne un exemple d'une géométrie assez curieuse.

La partie blanche est $I^{+}$, la partie grisée est $I^{-}$. On prend $u_{H}(W)=\ln W$ et $u_{L}(W)=-\frac{10}{\exp W}-W^{-0,8}$. D'autre part $\frac{p_{H}}{1-p_{H}} / \frac{p_{L}}{1-p_{L}}=1,01$.

REMARQUE 1. En situation normale, $\alpha_{L}$ appartient à $I^{+}$; en situation $S$, un contrat $\alpha_{L}$ profitable appartient à $T$.

En effet, notons $\left(W_{1}, W_{2}\right)$ les consommations prescrites par $\alpha_{L} . \mathrm{Si}$

$$
\frac{p_{H} u_{H}^{\prime}\left(W_{2}\right)}{\left(1-p_{H}\right) u_{H}^{\prime}\left(W_{1}\right)} \neq \frac{p_{L} u_{L}^{\prime}\left(W_{2}\right)}{\left(1-p_{L}\right) u_{L}^{\prime}\left(W_{1}\right)}
$$

les TMS des deux agents étant différents, il existe toujours une modification infinitésimale de $W_{1}$ et $W_{2}$ qui est plus attractive pour $L$ et moins attractive pour $H$. Si la contrainte de profit positif pour l'assureur de $L$ n'est pas serrée, 
FIGURE 3

Exemple de positionnement de $I^{+}, I^{-}$et $T$

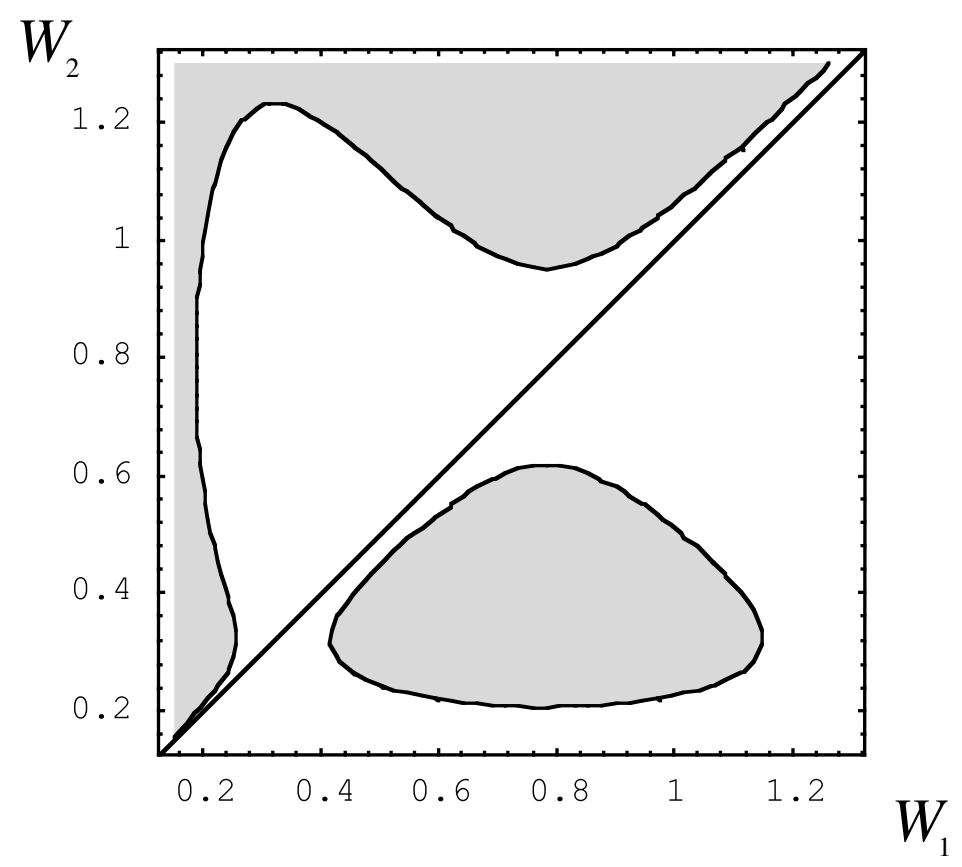

alors cette modification peut toujours être financée. L'inégalité (??) est donc incompatible avec l'existence d'un équilibre à profit positif.

Nous voyons ici une différence fondamentale entre la situation normale et la situation $S$. Dans la situation normale, les courbes d'indifférence des deux types se coupent franchement en tout contrat d'équilibre, ce qui ouvre la possibilité d'écrémage, ou encore d'attraction sélective d'un type. Dans la situation $S$, la tangence empêche ce type d'opération, et bloque le mécanisme de concurrence à la Bertrand, qui faire tendre les prix vers le bas.

Fixons les types $p_{H}$ et $p_{L}$. Il ne suffit pas que $u_{H}$ et $u_{L}$ représentent des attitudes face au risque différentes pour qu'on puisse bâtir des configurations d'équilibre qualitativement différentes de celle de RS. Tout d'abord, pour des types donnés (le risque et l'attitude face au risque), il n'existe pas forcément de partie du plan où la PCU est violée : le fait que les attitudes face au risque diffèrent n'est pas suffisant pour que $I^{-}$soit non vide. Ensuite, même si la PCU est violée dans une certaine partie de l'ensemble de consommation, la géométrie du problème, en confinant les offres dans $I^{+}$, peut permettre d'exclure que l'équilibre s'écarte qualitativement de celui de RS. Le fait que le voisinage de la droite à $45^{\circ}$ appartient à $I^{+}$(puisque $\frac{u_{K}^{\prime}\left(W_{2}\right)}{u_{K}^{\prime}\left(W_{1}\right)} \simeq 1$ ) rend facile le choix de $W$ et $d$ tels que la contrainte d'autosélection du haut risque ne porte que dans $I^{+}$. On prendra un $W$ quelconque, puis un $d$ suffisamment petit.

Ces remarques n'enlèvent rien au fait que si $u_{H}$ et $u_{L}$ sont données, $p_{H}$ et $p_{L}$ peuvent être choisies de façon à obtenir un ensemble $I^{-}$non vide : 
ThÉORÈme 2. Fixons $u_{H}$ et $u_{L}$ les utilités des types $H$ et des types $L$, respectivement. La situation est normale pour tout $\left(p_{L}, p_{H}, \lambda_{H}, \lambda_{L}, W\right.$, d) (avec $\left.p_{H}>p_{L}\right)$ si et seulement si $u_{H}$ est plus concave que $u_{L}$.

PREUVE : (Condition suffisante) Lorsque les agents ont la même fonction d'utilité, la PCU vient directement de la condition sur les risques, ou, si l'on veut, de l'ordre des ratios de probabilités. Lorsque les agents ont des fonctions d'utilité différentes, les ratios d'utilités marginales (les TMS sans les probabilités) peuvent fort bien être rangés en sens inverse des ratios de probabilités. Ainsi, si $u_{H}$ est plus concave que $u_{L}$, alors (PRATT [1964])

$$
\text { pour tous } W_{1}>W_{2}>0: \frac{u_{H}^{\prime}\left(W_{2}\right)}{u_{H}^{\prime}\left(W_{1}\right)} \geqslant \frac{u_{L}^{\prime}\left(W_{2}\right)}{u_{L}^{\prime}\left(W_{1}\right)}
$$

ce qui est une condition suffisante pour que $I^{+}$recouvre la partie de l'ensemble de consommation intéressant ce problème (c'est-à-dire sous la droite de pleine assurance). En effet, nous montrons au point 2 de la proposition 3 (indépendante de ce théorème) que $W_{1} \geqslant W_{2}$ : la sur-assurance avantageant relativement $H$, elle ne peut être efficace. Ce résultat n'est pas trivial dès lors que les attitudes face au risque diffèrent; sa démonstration nécessite la notion de contrats aléatoires, que nous avons préféré introduire en fin d'article par souci de lisibilité.

(Condition nécessaire) $\mathrm{Si} u_{L}$ n'est pas faiblement moins riscophobe que $u_{H}$, alors, en raison de la continuité de l'aversion pour le risque, il existe un intervalle $J$ où $u_{H}$ est strictement moins riscophobe que $u_{L}$. Il suffit maintenant de construire un problème d'assurance $\mathcal{P}$ dont tous les niveaux de consommation seront compris dans $J$. On choisit $W$ et $d$ tels que $[W-d, W] \subset J$. On cherche $p_{L}$ et $p_{H}$ (où $p_{L}<p_{H}$ ) tel que l'équilibre de Rothschild et Stiglitz existe, est séparateur, mais implique un profit positif sur le contrat offert au bas risque.

Choisir $p_{L}$ et $p_{H}$ a pour but d'assurer que $I^{-}$n'est pas vide. Comme nous l'avons déjà mentionné, même pour $p_{H}, u_{H}$ et $u_{L}$ fixés, le choix correct de $p_{L}$ est délicat puisqu'il influe à la fois sur les positions de la droite d'assurance actuarielle (donc sur $\alpha_{L}^{Z}$ ) et de $I^{-}$. En conséquence, il n'est pas clair en général si $\alpha_{L}$ appartient à $I^{-}$ou non.

Plus précisément, faire tendre $p_{L}$ vers $p_{H}$ élargit de façon monotone $I^{-}$qui s'approche de plus en plus près de la droite d'assurance complète, mais en même temps, $\alpha_{L}^{Z}$, que la contrainte d'autosélection de $H$ définit, converge vers $\alpha_{H}$ (qui appartient à la droite d'assurance complète) puisque les droites actuarielles se rapprochent de plus en plus. Il faut pour trancher déterminer si c'est $T$ ou $\alpha_{L}^{Z}$ qui s'approche le plus vite de la droite d'assurance complète.

La stratégie de démonstration est la suivante : a) nous fixons $p_{H}$ (ou cette probabilité nous est donnée). Il existe une symétrie entre $p_{H}$ et $p_{L}$ : c'est aussi bien cette dernière qui pourrait être fixée. Le choix est fait pour 
simplifier mais la généralité du raisonnement est évidente. b) Nous calculons la vitesse de convergence de :

$$
\left[\frac{p_{L}}{1-p_{L}}\right] /\left[\frac{p_{H}}{1-p_{H}}\right]
$$

vers 1 lorsque $p_{L}$ tend vers $p_{H}$ par valeurs inférieures. c) Nous calculons la vitesse de convergence de $\alpha_{L}^{Z}$ vers $\alpha_{H}$ en utilisant la contrainte d'autosélection. Cela constitue un premier pas pour calculer d) la vitesse de convergence de :

$$
\left[\frac{u_{H}^{\prime}\left(W_{2}\right)}{u_{H}^{\prime}\left(W_{1}\right)}\right] /\left.\left[\frac{u_{L}^{\prime}\left(W_{2}\right)}{u_{L}^{\prime}\left(W_{1}\right)}\right]\right|_{\left(W_{1}, W_{2}\right)=\alpha_{L}^{Z}\left(p_{L}\right)}
$$

vers 1 lorsque $p_{L}$ tend vers $p_{H}$ par valeurs inférieures.

Le fait que le ratio des ratios de probabilités (sic) (10) converge plus vite vers 1 que le ratio des ratios des utilités marginales (11) suffit à établir que pour tout $p_{L}$ suffisamment proche de $p_{H}, \alpha_{L}^{Z}$ se situe nécessairement dans $I^{-}$, de telle sorte que les assureurs obtiennent un profit strictement positif sur le contrat $\alpha_{L}$ qu'ils destinent au consommateur de bas risque.

a) La probabilité d'accident du haut risque est fixée à une valeur quelconque admissible $\left(0<p_{H}<1\right)$.

b) On a facilement :

(12) $\left[\frac{p_{L}}{1-p_{L}}\right] /\left[\frac{p_{H}}{1-p_{H}}\right]-1 \underset{p_{L} \uparrow p_{H}}{=}-\frac{p_{H}-p_{L}}{p_{H}\left(1-p_{H}\right)}+o\left(p_{H}-p_{L}\right)$

c) L'équation qui définit implicitement $\alpha_{L}^{Z}$ s'écrit ( $\phi$ étant la franchise, la prime vaut $\left.p_{L}(d-\phi)\right)$ :

$$
\begin{aligned}
& u_{H}\left(W-p_{H} d\right)= \\
& \left(1-p_{H}\right) u_{H}\left(W-p_{L} d+p_{L} \phi\right)+p_{H} u_{H}\left(W-p_{L} d-\left(1-p_{L}\right) \phi\right)
\end{aligned}
$$

Développons cette expression jusqu'au second ordre (par rapport à $\phi$ et $\left.p_{H}-p_{L}\right)$ au point $W-p_{H} d$

$$
\begin{gathered}
\text { (14) } p_{H} \times\left\{u_{H}\left(W-p_{H} d\right)+u_{H}^{\prime}\left(W-p_{H} d\right)\left[\left(p_{H}-p_{L}\right) d-\left(1-p_{L}\right) \phi\right]\right. \\
\left.+\frac{1}{2} u_{H}^{\prime \prime}\left(W-p_{H} d\right)\left[\left(p_{H}-p_{L}\right) d-\left(1-p_{L}\right) \phi\right]^{2}\right\} \\
+\left(1-p_{H}\right) \times\left\{u_{H}\left(W-p_{H} d\right)+u_{H}^{\prime}\left(W-p_{H} d\right)\left[\left(p_{H}-p_{L}\right) d+p_{L} \phi\right]\right. \\
\left.+\frac{1}{2} u_{H}^{\prime \prime}\left(W-p_{H} d\right)\left[\left(p_{H}-p_{L}\right) d+p_{L} \phi\right]^{2}\right\} \\
=u_{H}\left(W-p_{H} d\right)+o\left(p_{H}-p_{L}\right)^{2}+o\left(\phi^{2}\right)+o\left(\left(p_{H}-p_{L}\right) \phi\right)
\end{gathered}
$$


(tous les « $O »$ sont écrits aussi longtemps que l'ordre de $\phi$ reste indéterminé relativement à celui de $p_{H}-p_{L}$.) Après quelques simplifications, on obtient :

$$
\begin{aligned}
& \left(p_{L}-p_{H}\right) u_{H}^{\prime}(\cdot) d= \\
& \quad \begin{aligned}
u_{H}^{\prime}(\cdot)\left(p_{L}-p_{H}\right) \phi & +\frac{1}{2} p_{L}\left(1-p_{L}\right) u_{H}^{\prime \prime}(\cdot) \phi^{2} \\
& +o\left(p_{H}-p_{L}\right)^{2}+o\left(\phi^{2}\right)+o\left(\left(p_{H}-p_{L}\right) \phi\right)
\end{aligned}
\end{aligned}
$$

Le terme d'ordre supérieur du membre de gauche est le second, donc :

(16) $\phi \underset{p_{L} \uparrow p_{H}}{=}\left[\frac{2 d}{a_{H}\left(W-p_{H} d\right)}\right]^{1 / 2}\left[\frac{p_{H}-p_{L}}{p_{H}\left(1-p_{H}\right)}\right]^{1 / 2}+o\left(p_{H}-p_{L}\right)^{1 / 2}$

où $a_{H}$ est l'indice d'Arrow-Pratt d'aversion pour le risque de $u_{H}$.

d) Pour $K=H, L$ :

$$
\frac{u_{K}^{\prime}\left(W-p_{L} d-\left(1-p_{L}\right) \phi\right)}{u_{K}^{\prime}\left(W-p_{L} d+p_{L} \phi\right)}-1 \underset{\phi \downarrow 0}{=} a_{K}\left(W-p_{H} d\right) \phi+o(\phi)
$$

Donc :

$$
\begin{aligned}
& \frac{\left[\frac{u_{H}^{\prime}\left(W-p_{L} d-\left(1-p_{L}\right) \phi\right)}{u_{H}^{\prime}\left(W-p_{L} d+p_{L} \phi\right)}\right]}{\left[\frac{u_{L}^{\prime}\left(W-p_{L} d-\left(1-p_{L}\right) \phi\right)}{u_{L}^{\prime}\left(W-p_{L} d+p_{L} \phi\right)}\right]}-1 \underset{p_{L} \uparrow p_{H}}{=} \\
& {\left[a_{L}\left(W-p_{H} d\right)-a_{H}\left(W-p_{H} d\right)\right] \phi+o(\phi)}
\end{aligned}
$$

Rappelons que nous nous sommes placés dans $J$, où $L$ est localement plus riscophobe que $H$. Le coefficient de $\phi$ est donc strictement positif. La comparaison des vitesses de convergence vers 1 est maintenant possible.

Si $u_{H}$ est strictement moins concave que $u_{L}$, alors quels que soient $W, d$ et $p_{H}$, il existe une probabilité $p_{L}$ (où $p_{L}<p_{H}$ ) telle que $\mathcal{P}\left[N, p_{H}, u_{H}, \lambda_{H}, p_{L}, u_{L}, \lambda_{L}, W, d\right]$ soit en situation $S$. Supposons que $u_{H} n e$ soit pas plus concave que $u_{L}$. Notons $J$ l'ensemble non vide dans lequel l'agent $L$ est strictement plus riscophobe. $[W-d, W] \subset J$ est une condition suffisante pour que, quel que soit $p_{H}$, il existe une probabilité $p_{L}$ (ou une probabilité $p_{H}$ si l'on fixe $p_{L}$ ) conduisant à la situation $S$.

Ces résultats montrent que les hypothèses de RS peuvent être affaiblies pour ce qui regarde les aversions pour le risque, cependant leur résultat de profit nul est maintenu pour toutes les richesses et niveaux de sinistre si et seulement si les meilleurs risques sont aussi les moins riscophobes. Il y a néanmoins une robustesse locale de leurs prédictions dans le sens où les profits positifs n'apparaissent que si, toutes autres choses égales par ailleurs, donc pour des préférences données, les probabilités sont assez proches, ou si, pour des risques donnés, les différences d'aversion pour le risque sont suffisamment grandes. 


\subsection{Concurrence multidimensionnelle}

Jusqu'ici, l'asymétrie d'information portait sur deux données (risque et préférences), mais elles étaient parfaitement corrélées au sens où connaître l'une suffisait à connaître l'autre. Nous examinons dans cette section le cas multidimensionnel où les hauts et les bas risques peuvent avoir pour fonction d'utilité, soit $u$, soit $v$. On notera les types $H_{u}, H_{v}, L_{u}$ et $L_{v}$. L'étude de la concurrence permet des simplifications, puisqu'un assureur donné n'a pas à coordonner de menus complexes. Cela explique une complexité moindre que celle qu'on trouve dans les études de monopoles (par exemple, RocHET et CHONÉ [1998]).

Partons de $\alpha_{H}^{*}$ et prenons la contrainte résultant des deux contraintes d'autosélection de $H_{u}$ et de $H_{v}$. Sous cette contrainte d'autosélection mixte et sous la contrainte de profit positif ou nul, les contrats préférés par $L_{u}$, d'une part, et $L_{v}$, d'autre part, sont notés $\alpha_{L_{u}}$ et $\alpha_{L_{v}}{ }^{5}$. L'un au moins, disons $\alpha_{L_{u}}$ sans perte de généralité, réalise un profit nul ${ }^{6}$. Si l'autre lui est confondu (on définit alors $\alpha_{L} \equiv \alpha_{L_{u}}=\alpha_{L_{v}}$ ) et réalise un profit nul, alors nous dirons que nous sommes dans une situation normale généralisée, s'il réalise un profit strictement positif, nous sommes dans une situation $S$ généralisée.

Proposition 2. Soient $\lambda_{H_{u}}, \lambda_{H_{v}}, \lambda_{L_{u}}$ et $\lambda_{L_{v}}$ les proportions respectives des types $H_{u}, H_{v}, L_{u}$, et $L_{v}$.

1. $\alpha_{H}^{*}$ est pris à l'équilibre par une fraction non-nulle des $H$.

2. Dans la situation normale généralisée, tout $H$ prend $\alpha_{H}^{*}$ et tout $L$ prend $\alpha_{L}^{Z}$. L'équilibre existe si et seulement si $\lambda_{L_{u}}+\lambda_{L_{v}}$ est inférieur à un certain seuil.

3. Dans la situation $S$ généralisée, $L_{u}$ prend $\alpha_{L_{u}}$, alors que $\alpha_{L_{v}}$ est pris par $L_{v}$ et une fraction (positive ou nulle) des $H$. Cette fraction doit être comprise entre zéro et la valeur qui annule les profits réalisés par $\alpha_{L_{v}}$ sur L. L'équilibre existe si et seulement si $\lambda_{L_{u}}$ et $\lambda_{L_{v}}$ sont assez petits.

Preuve : 1. Ce point est analogue au point 2 de la proposition 1 , sachant que $N=+\infty$.

2. et 3. Les mécanismes concurrentiels conduisent à donner les meilleures assurances possibles aux $L$ sous contrainte de profit positif ou nul et sous contrainte que ce choix ne soit strictement dominant ni pour $H_{u}$, ni pour $H_{v}$. Les mélanges sont cependant possibles jusqu'à un certain point, ce qui autorise un certaine dispersion des $H$.

Concernant les conditions d'existence, les contraintes sur les distributions de types ont la même origine que dans RS : dès lors que le nombre de $L$ est assez grand, des contrats réunissant des bas risques et des hauts risques sont meilleurs pour les bas risques que $\alpha_{L}$ et restent finançables. Ces contrats éliminent le candidat à l'équilibre déterminé par les conditions nécessaires. On conclut dans ce cas à l'inexistence.

5. Il n'est pas nécessaire de supposer qu'un seul candidat existe pour chacun des bas risques. Pour alléger, nous en prenons simplement un si un choix est possible.

6. Ce cas est celui où $H_{u}$ restreint le plus, sur la droite actuarielle des $L$, les contrats possibles. Dans ce cas, selon l'analyse standard de RS, le meilleur contrat de $L_{u}$ est bien le contrat de profit nul $\alpha_{L_{u}}$. 
Une configuration d'équilibre possible est donc la suivante. Les haut risques de forte aversion pour le risque prennent une assurance complète actuarielle. Les hauts risques de faible aversion pour le risque et les bas risques de forte aversion pour le risque prennent un contrat qui réalise un profit positif, éventuellement strictement positif, en moyenne. Les bas risques de faible aversion pour le risque prennent une assurance partielle actuarielle.

\section{L'assurance aléatoire}

\subsection{Principe}

Alors qu'un contrat d'assurance « normal » associe à toute contingence (le niveau de perte) un transfert, le contrat aléatoire associe un transfert décidé par une loterie (éventuellement dégénérée). Cet espace des stratégies élargi par convexification (voir PRESCOTT et TOWNSEND [1984]) permet de renforcer l'incitation à un meilleur effort dans le cas de l'aléa moral, ou de limiter le rationnement de certains agents en antisélection.

L'assurance aléatoire n'est apparemment pas observée en pratique, peutêtre en raison du soupçon de manipulation. Pourtant, il existe un moyen simple de simuler une loterie. Il suffit que le contrat conditionne le remboursement non seulement à la perte monétaire, mais aussi à certaines circonstances matérielles de l'accident. Pour peu que ces circonstances soient elles-mêmes aléatoires, elles permettent très simplement de soutenir une forme de loterie. Par ailleurs, la randomisation n'a pas besoin d'être complexe (section 4.2).

La structure du jeu concurrentiel est conservée : la séquence des coups, le degré et la durée des engagements, la notion d'équilibre, etc., ne sont pas touchés par cette possibilité. En particulier, l'incertitude des termes du contrats n'étant levée qu'après éventuelle acceptation par l'assuré, les contrats aléatoires en stratégies pures se distinguent bien des stratégies mixtes. Il reste à savoir si la loterie est tirée avant ou après l'accident. En aléa moral, cela fait une différence puisque les incitations à la prudence en dépendent, mais en antisélection, où la perte est exogène, les allocations dans les deux cas sont les mêmes.

Prescott et Townsend [1984] et ArnotT et Stiglitz [1988] l'ont montré, l'assurance aléatoire n'a pas d'intérêt dans le modèle original de RS. Remplaçons toute clause de remboursement aléatoire par un remboursement qui conduit l'agent à consommer l'équivalent certain de la consommation aléatoire prévue par la loterie. Or les deux agents ayant la même attitude face au risque, ce remboursement certain est le même pour tous les deux ; l'opération permet à l'assureur d'augmenter ses profits sans aucun affaiblissement du mécanisme d'autosélection.

Quand les attitudes face au risque des individus ne sont pas identiques, cette réduction n'est pas possible. Cela peut être exploité de la manière suivante. 
En mettant un peu d'aléa dans le contrat de $L$, tout en améliorant la qualité moyenne du remboursement, il est possible dans certaines conditions d'améliorer le contrat déterministe que $L$ recevrait sans attirer $H^{7}$.

\subsection{Structure des assurances aléatoires}

Nous adaptons certains résultats de LANDSBERGER et MEILIJSON [1999, dans la suite LM] à la concurrence montrant que la complexité de la randomisation est limitée. Une autre propriété intéressante est que les supports des loteries sont ordonnés. Plus il est vraisemblable, étant donnée la perte observée, qu'un agent soit un haut risque, et moins la consommation est élevée. Cela généralise le fait connu dans RS qu'il n'est pas offert de sur-assurance à l'équilibre. Notre démonstration met l'accent sur les absences de possibilités d'arbitrage qui sont nécessaires à la maximisation de son programme par l'assureur.

Proposition 3 (LM). 1. La réunion des supports des loteries du contrat du bas risque forme un ensemble dont le cardinal ne dépasse pas quatre.

2. Pour le bas risque, le support de la loterie en cas d'accident est inférieur au support de la loterie en l'absence d'accident.

PreUVE : 1. À l'équilibre, l'offre faite au bas risque maximise son espérance d'utilité sous les contraintes que le haut risque ne soit pas attiré (20) et que le profit soit positif (21). Formellement, dans le cas où le contrat de $H$ est actuariel mais l'argument est général, le contrat de $L$ est solution du programme suivant, où les variables de choix sont des loteries (c'est-à-dire les consommations aléatoires) $\tilde{W}_{1}, \tilde{W}_{2}$ :

$$
\max _{\tilde{W}_{1}, \tilde{W}_{2}}\left(1-p_{L}\right) E u_{L}\left(\tilde{W}_{1}\right)+p_{L} E u_{L}\left(\tilde{W}_{2}\right)
$$

sous les contraintes

$$
\begin{gathered}
\left(1-p_{H}\right) E u_{H}\left(\tilde{W}_{1}\right)+p_{H} E u_{H}\left(\tilde{W}_{2}\right) \leqslant u_{H}\left(W-p_{H} d\right) \\
\left(1-p_{L}\right) E \tilde{W}_{1}+p_{L} E \tilde{W}_{2} \leqslant W-p_{L} d
\end{gathered}
$$

Le programme se résout dans le simplexe des probabilités de chaque loterie. Le programme est donc linéaire et a quatre contraintes (la masse de probabilité doit valoir 1). La théorie de la programmation linéaire indique donc que quatre probabilités au plus sont strictement positives.

2. Soit $W_{1}$ (resp. $\left.W_{2}\right)$ une consommation du support de $\tilde{W}_{1}$ (resp. $\tilde{W}_{2}$ ). Nous raisonnons par l'absurde. Supposons que $W_{1}<W_{2}$ et notons $q_{1}$ et $q_{2}$ les probabilités conditionnelles (strictement positives) de ces consommations,

7. ViLleneuve [1996] discute ce point, et pointe en particulier une (légère) erreur dans ARNOTT et STIGLITZ [1987], un peu optimiste quant au pouvoir des assurances aléatoires. Intuitivement, si $L$ a vraiment peu d'aversion pour le risque, alors il n'y a pas beaucoup de gain à espérer de cette opération, puisqu'en fait il souffre peu de n'avoir qu'une assurance partielle. À la limite, un bas risque neutre au risque n'a rien à gagner de la randomisation puisque l'antisélection ne lui faisait rien perdre. 
respectivement, en l'absence de sinistre et en cas de sinistre. Autrement dit, $q_{1}$ représente la probabilité de $W_{1}$ dans la loterie $\tilde{W}_{1}$. Montrons que $q_{1}$ et $q_{2}$ peuvent être changées de telle façon que la contrainte de profit positif reste vérifiée, et que la contrainte d'autosélection du haut risque soit relâchée.

Introduisons $W_{1}$ dans le support de $\tilde{W}_{2}$ et $W_{2}$ dans le support de $\tilde{W}_{1}$ de manière que l'utilité du bas risque ne soit pas modifiée s'il prend ce contrat. Soit un réel $\varepsilon>0 ; q_{1}$ est remplacé par $q_{1}-\varepsilon$ et la probabilité $\varepsilon$ est transférée à l'atome $W_{2}$ dans $\tilde{W}_{1}$. Globalement, la probabilité de $W_{1}$ a été diminuée de $\left(1-p_{L}\right) \varepsilon$. Cette masse de probabilité est incorporée à $\tilde{W}_{2}$ : $\frac{1-p_{L}}{p_{L}} \varepsilon$ est ajoutée au poids de $W_{1}$ dans $\tilde{W}_{2}$. Pour conserver les masses, cette dernière probabilité conditionnelle est soustraite de $q_{2}$.

Pour peu que $\varepsilon$ soit assez petit, toutes ces opérations sont licites : il n'y a pas de masses négatives, et les sommes des probabilités conditionnelles des deux loteries valent 1. Elles sont neutres pour l'assureur si son client reste bien le bas risque, et pour le bas risque puisqu'il reste confronté à la même loterie globale, malgré les modifications des loteries partielles. La perception de la nouvelle loterie par le haut risque est changée. Pour le voir, limitons nous à la partie de la loterie qui a été modifiée. Le haut risque observe une détérioration au sens de la dominance stochastique au premier ordre et la variation d'utilité de $H$ vaut :

$$
\underbrace{\left\{\frac{p_{H}\left(1-p_{L}\right)}{p_{L}}-\left(1-p_{H}\right)\right\}}_{>0}(\underbrace{u_{H}\left(W_{1}\right)-u_{H}\left(W_{2}\right)}_{<0}) \varepsilon
$$

Le facteur $\varepsilon$ est arbitraire et donne l'échelle.

\subsection{Exclure les assurances aléatoires à profit positif}

L'assurance aléatoire et les profits positifs semblent devoir s'exclure mutuellement : l'origine des profits positifs, c'est la plus grande aversion pour le risque du bas risque, alors que l'origine de l'utilité de l'assurance aléatoire, c'est la plus faible aversion pour le risque du bas risque. Cependant, les aversions pour le risque ne sont pas nécessairement ordonnées globalement. Nous proposons donc une condition qui est suffisante pour qu'on puisse exclure la présence d'un contrat d'assurance aléatoire profitable.

Proposition 4. Si les aversions pour le risque $a_{H}$ et $a_{L}$, supposées continues, de $u_{H}$ et de $u_{L}$ se croisent une fois au plus, alors à l'équilibre, l'assurance aléatoire et le profit strictement positif s'excluent mutuellement.

Le croisement unique qui est invoqué ici s'entend ainsi : il existe un réel $W_{0}$ (éventuellement infini) tel que :

$$
a_{H}(W)<a_{L}(W) \Rightarrow W<W_{0} \text { et } a_{H}(W)>a_{L}(W) \Rightarrow W>W_{0}
$$

$$
a_{H}(W)>a_{L}(W) \Rightarrow W<W_{0} \text { et } a_{H}(W)<a_{L}(W) \Rightarrow W>W_{0}
$$


Cette définition n'exclut donc pas qu'il y ait plusieurs (et même une infinité) de points de tangence. Elle équivaut à supposer que la fonction $u_{L}^{-1} o u_{H}$ ne change strictement de courbure qu'une fois au plus.

PREUVE : Supposons que $\alpha_{L}$ soit profitable et aléatoire pour l'un des états au moins. Nous utilisons deux résultats :

1) $\forall W_{1} \in \operatorname{supp} \tilde{W}_{1}, W_{2} \in \operatorname{supp} \tilde{W}_{2}: \frac{p_{H} u_{H}^{\prime}\left(W_{2}\right)}{\left(1-p_{H}\right) u_{H}^{\prime}\left(W_{1}\right)}=\frac{p_{L} u_{L}^{\prime}\left(W_{2}\right)}{\left(1-p_{L}\right) u_{L}^{\prime}\left(W_{1}\right)}$;

2) $\forall W \in \operatorname{supp} \tilde{W}_{1} \cup \operatorname{supp} \tilde{W}_{2}: a_{L}(W) \geqslant a_{H}(W)$.

Le premier point découle de la remarque 1. Le second est montré par l'absurde. Si $a_{L}(W)<a_{H}(W)$ pour l'une des consommations du support de l'une des loteries, prenons un réel $\varepsilon>0$; changeons la consommation $W$ en $W-\varepsilon+\pi$ avec une probabilité 0,5 et $W+\varepsilon+\pi$ avec une probabilité 0,5 , où $\pi$ est tel qu'il compense exactement $L$ de la perte d'utilité provoquée par l'aléa :

$$
0,5 u_{L}(W+\varepsilon+\pi)+0,5 u_{L}(W-\varepsilon+\pi)=u_{L}(W)
$$

La continuité de l'aversion pour le risque permet de choisir $\varepsilon$ assez petit pour que $a_{L}$ soit strictement plus petit que $a_{H}$ sur $[W-\varepsilon+\pi$, $W+\varepsilon+\pi]$, et assez petit pour que le profit de l'assureur reste positif. En conséquence, $\pi$ ne peut pas compenser le haut risque (PRATT [1964]) et la contrainte d'autosélection est bien relâchée.

Montrons maintenant la proposition. Supposons que le contrat du type $L$ spécifie une loterie non dégénérée en cas d'accident, et fixons $W_{1}$ une consommation prévue si l'accident ne survient pas. Le cardinal de supp $\tilde{W}_{2}$ est 2 ou 3 (voir proposition 3 ). D'après 1 ), les $W_{2}$ correspondantes sont solution de :

$$
\frac{u_{H}^{\prime}\left(W_{2}\right)}{u_{L}^{\prime}\left(W_{2}\right)}=\frac{p_{L}\left(1-p_{H}\right)}{\left(1-p_{L}\right) p_{H}} \frac{u_{H}^{\prime}\left(W_{1}\right)}{u_{L}^{\prime}\left(W_{1}\right)}
$$

Donc $u_{H}^{\prime}(\cdot) / u_{L}^{\prime}(\cdot)$ doit ne pas être strictement monotone, et change son sens de variation au moins une fois. (Si cette fonction est constante sur l'enveloppe convexe du support, alors la randomisation est inutile puisque les aversions pour le risque seraient identiques). Par ailleurs, $a_{L}(W) \leqslant$ $(<) a_{H}(W)$ si, et seulement si, $u_{H}^{\prime}(\cdot) / u_{L}^{\prime}(\cdot)$ est (strictement) décroissante en $W$. Or 2) interdit les points où $u_{H}^{\prime}(\cdot) / u_{L}^{\prime}(\cdot)$ est strictement croissante. Cet ensemble de conditions nécessaires n'est pas réuni si les aversions pour le risque se croisent une fois au plus. 


\section{Discussion}

La condition de croisement unique dans le modèle de RS et ses dérivés a pour mérite principal de faciliter la caractérisation des équilibres ${ }^{8}$. Elle n'a pas de mérite supérieur en ce qui concerne son réalisme. Dans notre projet, où nos raisonnements doivent se passer d'elle, il est important de noter que la concurrence apporte tout de même des simplifications dans l'analyse, puisque les assureurs sont principalement préoccupés des coûts, et n'ont pas à arbitrer la réduction des rentes informationnelles et la conservation des incitations. Ainsi, notre généralisation montre comment les intuitions du modèle à deux types se transcrivent dans un modèle plus complet, véritablement multidimensionnel (voir section 3.3).

Le concept d'équilibre de Rothschild et Stiglitz n'est pas sans défaut, en raison notamment de l'hypothèse que les assureurs n'offrent qu'un contrat (on peut aussi l'interpréter comme l'obligation que tout contrat soit bénéficiaire). Dans la concurrence dite en menus de contrats (HAHN [1978]), considérée comme plus réaliste, les assureurs peuvent offrir autant de contrats qu'ils le souhaitent (ils offrent des menus, ou des tarifs, voir HENRIET et RocHET [1988]), et l'équilibre est un équilibre de Nash.

Toute paire de contrats qui n'est pas un optimum de Pareto sous contraintes informationnelles ne peut pas être un équilibre de Hahn (sinon une offre supérieure au sens de Pareto faite par un assureur attirerait toute la clientèle). Autrement dit, tout équilibre de Hahn en stratégie pure, s'il existe, est un optimum de second rang. Or, dans la situation $S$, toutes les offres optimales donnent au moins l'utilité de son contrat actuariel à $H$. En effet, la seule manière de «rendre » aux assurés les profits sur $L$ consiste à soulager la contrainte que subit $L$, donc à offrir une meilleure assurance à $H$, laquelle est nécessairement déficitaire. Mais dans une situation où il faut accepter des pertes sur le haut risque et réaliser des gains sur le bas risque, tout assureur est incité à se spécialiser dans l'assurance des bas risques, seuls assurés profitables. Ce comportement sélectif n'est pas possible à l'équilibre.

En conséquence, dans la situation $S$, il n'existe pas d'équilibre de Nash en stratégies pures du jeu de Hahn. Les équilibres en stratégies mixtes ne pouvant pas être efficaces ${ }^{9}$, l'intervention du gouvernement en ces matières trouve un fondement sûr. L'efficacité dans la situation $S$ requiert des subventions croisées entre types quelle que soit la distribution des types. Jusqu'alors, il était apparu aux théoriciens que certaines distributions des risques justifiaient une intervention publique, quand l'équilibre de RS n'existe pas ou lorsqu'il est inefficace, autrement dit quand les types rationnés sont assez nombreux. La conclusion normative que nous proposons est que certaines

8. Voir, par exemple, DiONNE et DOHERTY [1991] pour une revue de la littérature.

9. DASGUPTA et MASKIn [1986] ont montré que l'équilibre de Hahn existait toujours lorsqu'on autorise les stratégies mixtes. Appliquer leur méthode dans notre variante du modèle de RS est délicat. SiMON et ZAME [1990] offrent un théorème puissant qui permet de conclure à l'existence : il suffit de ne pas choisir a priori la manière dont les agents se répartissent entre les offres optimales qu'ils reçoivent ; malgré la discontinuité des paiements du jeu, l'hémi-continuité supérieure de l'ensemble des choix possibles pour les joueurs permet l'application du théorème. 
hétérogénéités justifient toujours de tels transferts, quel que soit la distribution des types. Le moyen le plus simple reste l'assurance obligatoire partielle, réalisant le transfert nécessaire, pourvu qu'elle soit complétée par un marché pour l'assurance complémentaire.

\section{- Références bibliographiques}

ARMStrong M. (1996). - «Multiproduct Nonlinear Prices », Econometrica, 64, p. 51-75.

ARnotT R., Stiglitz J.E. (1988). - «Randomization with Asymmetric Information », Rand Journal of Economics, 19, p. 344-362.

Chassagnon A. (1996). - « Sélection adverse : modèle générique et applications », Thèse de doctorat, EHESS.

Chiappori P.-A., Macho I., Rey P., Salanié B. (1994). - « Repeated Moral Hazard: The Role of Memory, Commitment, and the Access to Credit Markets », European Economic Review, 38, p. 1527-1553.

DAsGuPTA P., MASKIN E. (1986). - « The Existence of Equilibrium in Discontinuous Economic Games, II: Applications », Review of Economic Studies, 53, p. 27-41.

De Meza D., WebB D. (2000). - «Advantageous Selection in Insurance Markets », London School of Economics, Mimeo.

Dionne G., Doherty N.A. (1991). - «Adverse Selection in Insurance Markets: A Selective Survey », in Contributions to Insurance Economics, Dionne G. (éd.), Kluwer Academics.

Doherty N.A., Schlesinger H. (1983). - « Optimal Insurance in Incomplete Markets », Journal of Political Economy, 91, p. 1045-1054.

FAGART M.-C. (1996). - «Concurrence en contrats, anti-sélection et structure d'information », Annales d'Économie et Statistique, 43, p. 1-28.

Fluet C., Pannequin F. (1997). - «Complete versus Incomplete Insurance Contracts Under Adverse Selection with Multiple Risks », Geneva Papers on Risk and Insurance Theory, 22, p. 81-101.

Guesnerie R., LAFFont J.-J. (1984). - «A Complete Solution of a Class of Principal Agent Problems with an Application to the Control of Self-Managed Firms », Journal of Public Economics, 25, p. 329-369.

Hahn F. (1978). - «On Equilibrium with Market-Dependent Information», Quantitative Wirtschaftforschung.

HenRIET D., Rochet J.-C. (1988). - «Équilibres et optima sur les marchés d'assurance : Une illustration des phénomènes d'antisélection », in Essais en l'honneur d'Edmond Malinvaud, Economica, Paris.

Jullien B., Salanié B., Salanié F. (2000). - « Screening Risk-Averse Agents Under Moral Hazard », document de travail CREST 2000-41.

Koenl P.-F., Villeneuve B. (2001). - « Complementarity and Substitutability in Multiple Risk Insurance Markets », International Economic Review, 42, p. 245-266.

LAFFont J.-J., Rochet J.-C. (1988). - « Stock Market Portfolios and the Segmentation of the Insurance Market », Scandinavian Journal of Economics, 90, p. 435-447.

Landsberger M., Meilijson I. (1999). - « A General Model of Insurance under Adverse Selection », Economic Theory, 14, p. 331-352.

PRATT J. (1964). - « Risk Aversion in the Small and in the Large », Econometrica, 32, p. 122-136.

Prescott E.C., Townsend R.M. (1984). - « Pareto Optima and Competitive Equilibria with Adverse Selection and Moral Hazard », Econometrica, 52, p. 21-45.

RILEY J.G. (1979). - « Informational Equilibrium », Econometrica, 47, p. 331-359.

Rochet J.-C., CHONÉ P. (1998). - « Ironing, Sweeping, and Multidimensional Screening », Econometrica, 66, p. 783-826. 
Rothschild M., Stiglitz J.E. (1976). - « Equilibrium in Competitive Insurance Markets: An Essay on the Economics of Imperfect Information », Quarterly Journal of Economics, 90, p. 629-649.

Simon L.K., Zame W.R. (1990). - «Discontinuous Games and Endogenous Sharing Rules », Econometrica, 58, p. 861-872.

SMART M. (2000). - « Competitive Insurance Markets with Two Unobservables », International Economic Review, 41, p. 153-169.

VILleneuve B. (1996). - «Essais en économie de l'assurance», Thèse de doctorat, EHESS.

WAMBACH A. (1997). - «Equilibrium in competitive insurance markets-revisited », papier présenté au Congrès de la Société d'Économétrie, Toulouse. 